REVIEW

\title{
Tuning noninvasive brain stimulation with MRI to cope with intersubject variability
}

\author{
Annegret Habich ${ }^{\mathrm{a}}$, Santiago Canals ${ }^{\mathrm{b}}$, and Stefan Klöppe/ ${ }^{\mathrm{a}, \mathrm{c}, \mathrm{d}}$
}

\begin{abstract}
Purpose of review
The review aims at highlighting the additional benefit that can be gained from combining noninvasive brain stimulation as well as repetitive sensory stimulation protocols with MRI techniques to account for the intersubject variability observed in those treatments. Potentially, this should help to identify predictive patterns in the individual receptiveness to the treatment.
\end{abstract}

\begin{abstract}
Recent findings
Knowledge about the underlying physiological principles of excitability changes as induced by noninvasive brain stimulation or repetitive sensory stimulation is accumulating, revealing strong associations with plasticity processes at the synaptic level. In this context, MRI techniques, such as magnetic resonance spectroscopy and functional MRI, emerged as valuable tools for the qualitative assessment of baseline states and induced changes. Those physiological readouts can help explain the interindividual heterogeneity found in behavioural and/or clinical responses to the specific stimulation protocols. This knowledge will eventually translate, first, into the preliminary classification of study participants into treatment groups according to their neurophysiological baseline state and expected responses to a particular stimulation. Subsequently, this should also aid the optimization of stimulation protocols according to the classification outcome, resulting in retuned protocols for particular groups of study participants.
\end{abstract}

\section{Summary}

The consistent MRI-based monitoring of stimulation effects in the neural network promises a considerable gain for the customization of intervention protocols with improved therapeutic potential and rehabilitative predictions.

\author{
Keywords \\ intersubject variability, long-term potentiation, MRI, repetitive sensory stimulation, transcranial direct \\ current stimulation
}

\section{INTRODUCTION}

Since time immemorial, humans have sought to boost their cognitive abilities. For many years, nootropic chemical substances were the means of choice, but over the past few decades, noninvasive brain stimulation (NIBS) techniques, including transcranial direct current stimulation (tDCS), stirred the scientific community. Positive impacts on vocabulary learning [1], multitasking performance [2], and visual search efficiency [3"'] number among the favourable effects attributed to NIBS. But also, more critical voices were raised [4], adducing ethical concerns [5] and the possibility of cognitive trade-offs [6]. Accumulating observations highlight the large heterogeneity of the responses across study participants [7], with some of them even addressing the possibility of a mere placebo effect [8]. In a constantly growing body of work, apart from promising neural enhancement in healthy individuals, tDCS is applied as a treatment for behavioural and neurological disorders nonetheless $\left[9,10,11^{"}\right]$.

\footnotetext{
${ }^{a}$ Department of Psychiatry and Psychotherapy, University Medical Center Freiburg, Freiburg, Germany, 'Instituto de Neurociencias, Consejo Superior de Investigaciones Cientificas, Universidad Miguel Hernández, Sant Joan d'Alacant, Spain, ${ }^{\circ}$ Center of Geriatrics and Gerontology Freiburg and ${ }^{\mathrm{d} D e p a r t m e n t}$ of Neurology, Freiburg Brain Imaging, University Medical Center Freiburg, Freiburg, Germany

Correspondence to Annegret Habich, MSc, Department of Psychiatry and Psychotherapy, University Medical Center Freiburg, Hauptstraße 5 , 79104 Freiburg, Germany. Tel: +49 178 9388316; e-mail: annegret.habich@uniklinik-freiburg.de
}

Curr Opin Neurol 2016, 29:000-000

DOI:10.1097/WCO.0000000000000353 


\section{KEY POINTS}

- Effects, which are induced by brain and sensory stimulation, coincide only partially with plasticity processes observed at the synaptic level.

- Magnetic resonance-based techniques can improve the understanding of metabolic and connectivity changes in the brain network elicited by NIBS or rSS.

- The characterization and quantification of the physiological changes induced by noninvasive brain stimulation can help account for the observed large intersubject variability in treatment effects and furthermore to predict the individual responsiveness to the treatment.

\section{NEUROPHYSIOLOGICAL CHARACTERISTICS OF NONINVASIVE BRAIN STIMULATION AND REPETITIVE SENSORY STIMULATION EFFECTS}

Insofar, there is a general consensus that as a neuromodulatory technique tDCS produces polaritydependent changes in cortical excitability. Rather than eliciting action potentials, tDCS introduces a tonic depolarization or hyperpolarization to the membrane resting potential of the neurons in the targeted brain area. Similarly, repetitive sensory stimulation (rSS) protocols that stimulate peripheral sensory systems have been considered to produce network correlates of synaptic plasticity in a frequency-dependent manner, with higher frequencies of stimulus presentation producing long-term potentiation (LTP)-like effects, whereas lower frequencies induce long-term depression (LTD)-like effects [12-14]. Both processes, LTP and LTD, are currently considered as key synaptic underpinnings of learning and memory.

It has been demonstrated that tDCS in vivo modulates short as well as long-term types of synaptic plasticity $\left[15,16^{\prime \prime}\right]$, paired-pulse facilitation (PPF), and LTP in particular. Despite the conflictive results regarding the exact effects of tDCS on PPF, which might be attributed to the different recording sites and distinct neurotransmitter release probabilities in these brain regions, the impact of the stimulation on the presynaptic sites is noteworthy. Moreover, an additional effect of tDCS on the postsynaptic sites was implicated in a study by Rohan and colleagues [16"'], wherein, subsequent to tDCS, the induction of LTP in acutely prepared brain slices was facilitated. This was reflected in a larger slope and amplitude of field potentials after tDCS as compared with the sham condition. This effect on synaptic plasticity, which was dose dependent and lasted for $24 \mathrm{~h}$, is the most prominent link between
tDCS and LTP. This labelling already implicates a connection to plasticity processes on the cellular level. However, the inflationary use of these terms is not always justified, given that the exact nature of the likeness is not documented in all studies. Accordingly, the existing criteria for the definition of LTP at the synapse, more specifically persistence, input specificity, associativity, and cooperativity, could be employed as a starting point to justify the choice of terminology on the network level while also entailing separate analyses of network stimulation effects. Finding a bridge between local plasticity processes observed at the cellular level and the global network behaviour might provide researchers with crucial hints as to the underlying neurophysiological factors for the observed high interindividual variability [17-19], which impedes the efficacy and reliability of common tDCS paradigms [20,21"'].

To what extent are NIBS and rSS effects mediated by synaptic plasticity processes? To answer this question will require additional experimental work on animal models combining simultaneous measurements of neuronal circuits at meso and macroscopic levels. Some clues, however, can be found by following classic neurophysiological definitions of synaptic plasticity.

Among the array of characteristics attributed to LTP, the persistence of the induced changes is probably the most sought after in a clinical context. In clinical settings, this has been most commonly monitored with regard to amplitude changes of motor-evoked potentials (MEPs) [22,23], often derived from transcranial magnetic stimulation, prior to, during and at several time points after the stimulation period, thus tracking when the stimulation-induced effects would return to their baseline levels. Single tDCS sessions, lasting for 10-20 min, produced increased MEP amplitudes up to $90 \mathrm{~min}$ after terminating the stimulation [20]. Furthermore, tDCS-induced effects from repeated application over the course of 5 consecutive days were still detected at follow-up examinations 4 weeks after the stimulation [24].

Although on the synaptic level, input specificity, cooperativity, and associativity are additional aspects that characterize LTP processes, to date, no techniques exist to reliably acknowledge their presence on the systems level in humans, mainly owing to the larger stimulated areas during NIBS and rSS as compared with the more focal points in classic invasive LTP and LTD-inducing stimulation protocols. Alternatively, functional network connectivity could be assumed to be an approximation to the combined definitions of input specificity and associativity to the extent that excitability changes are 
not restricted to the area directly beneath the electrodes but spread across different brain regions. Nor does this spreading proceed in an unspecific manner but it occurs along functional pathways instead, consequently rudimentarily modelling the synaptic concepts on a larger scale.

Although electroencephalographic recordings have been employed for reconstructing changes in functional connectivity patterns by means of neural synchronization in different frequency bands $[25,26]$, functional MRI (fMRI) has emerged as the foremost technique in studies of this kind.

Studies like the one conducted by Meinzer and colleagues [27], who could associate a reduced activity in the ventral inferior frontal gyrus with an improved behavioural performance in picture naming during task-related fMRI, illustrate the power of combining brain stimulation and neuroimaging techniques. Such studies are comparatively scarce, with the vast majority of research carried out using resting state fMRI and focussing on established functional networks [28-30]. But even when tDCS is applied in the resting state, it has been suggested that it primes networks for their differential recruitment in future tasks [31].

To what extent and in which direction excitability changes appear in specified networks depends on the electrode montage, unilaterally or bilaterally [32], the polarity, cathodal or anodal [33], and the stimulated cortical area. Furthermore, despite the adherence to the same parameters, different outcomes have been reported. For instance, Polanía and colleagues [33] reported a boost in local functional connectivity triggered by cathodal tDCS, whereas anodal tDCS affected functional connectivity at longer distances, whereas Amadi and colleagues [34] only found an increase in interhemispheric functional connectivity induced by cathodal tDCS, whereas neither sham nor anodal stimulation took any effect. Notwithstanding the diverging results, there is a common agreement as to the overall modulatory power of tDCS on the network connectivity of distinct, long-range functional networks with matching results derived from blood oxygen level-dependent signal and arterial spin labelling [35].

Owing to the historical studies of the N-methyl-D-aspartate receptor (NMDAR)-dependent LTP along the Schaffer collaterals in the hippocampus, the involvement of the aforementioned receptor is usually required when referring to LTP-like changes. It acts as a coincidence detector that recognizes the paired activity at pre and postsynaptic sites, which instigates lasting forms of synaptic plasticity by strengthening the synaptic contact. Several studies on rat hippocampi $\left[16^{-\prime}, 36\right]$ as well as in human volunteers [37] have demonstrated that the enhanced excitability induced by direct current stimulation and tDCS, respectively can be blocked by the application of NMDAR antagonists. This demonstrated the similarity between cellular and network plasticity processes, pertaining to the early phase of LTP induction, which mainly relies on the regulation of glutamate receptor activity and their insertion into the postsynaptic membrane. Additionally, late effects, which rely on gene expression and protein synthesis, were attested after the stimulation period. Fritsch and colleagues [38] could only show a heightened brain-derived neurotrophic factor (BDNF) secretion and a simultaneously increased activation of tropomyosin receptor kinase B when direct current stimulation and lowfrequency synaptic activation were combined; the epigenetic enhancement of $B D N F$ gene expression could be related to an exclusive tDCS protocol in the animal model elsewhere [39"]. In accordance with the observed absence of LTP effects after stimulation in BDNF knockout mice, the Val66Met polymorphism of the BDNF gene in humans has an impact on stimulation effects $\left[40^{\circ}\right]$. More specifically, Met carriers exhibited a greater corticospinal excitability and produced larger MEP responses to transcranial magnetic stimulation after stimulation as compared with Val/Val homozygotes.

Similarly, baseline $\gamma$-amino butyric acid (GABA) levels measured by magnetic resonance spectroscopy (MRS) can predict motor learning insofar as higher baseline concentrations are indicative of a lower stimulation gain [41]. Similar correlations emerged during rSS [42"-]. Therein, local GABA concentrations measured prior to the stimulation explained $60 \%$ of the variance in a tactile learning task even though no alterations in the corresponding neurotransmitter concentration substantiated after the treatment. Rather than quantifying neurotransmitter concentrations at individual synapses, MRS can be used to evaluate the levels of various inhibitory and excitatory neurometabolites in larger brain regions. Thus, it has been demonstrated that, contrary to either sham or cathodal tDCS, anodal stimulation reduces GABA concentrations locally in the left primary motor cortex M1 in healthy controls $\left[41,43^{-"}\right]$ as well as in the ipsilesional M1 in stroke patients [44]. These results coincide with the current notion that anodal tDCS leads to an increase of excitability, whereas a decrease in excitability is observed upon cathodal tDCS. Apart from the reduction of inhibitory neurotransmitters in the neural network, anodal tDCS also operates in the opposite direction, increasing excitatory neurotransmitter concentrations. This has been demonstrated for glutamatergic metabolites, glutamate 
and glutamine, as well as for combined N-acetylaspartate and $\mathrm{N}$-acetylaspartylglutamate $\left[45,46^{-1}\right]$. Those changes were restricted to the areas beneath the anodal stimulation electrode, whereas no augmentation appeared in homologous regions in the contralateral hemisphere.

Evidence for the multiplicity of tDCS effects on different physiological levels, not all being tantamount to conventional synaptic LTP, can be gathered from schizophrenia patients. The latter exhibit demonstrably decreased NMDAR levels [47] and decreased GABA transmission [48"], whereas tDCS still enhanced their working memory performance, presumably by restoring gamma oscillations [49"].

\section{MRI TECHNIQUES FOR THE SYSTEMATIC SURVEILLANCE OF NONINVASIVE BRAIN STIMULATION EFFECTS}

A pioneer study combining simultaneously fMRI with intracranial electrophysiological recordings and deep brain stimulation in rats was conducted to investigate the influence of local synaptic plasticity on long-range functional connectivity in the brain [50-52]. In this work, induction of LTP by high-frequency stimulation of the perforant pathway connecting the medial entorhinal cortex with the dorsal hippocampus resulted in brain-wide reorganization of neuronal networks. More specifically, after LTP stimulation and during several hours afterward, the authors found enhanced functional coupling of the hippocampal formation with the prefrontal cortex and the mesolimbic dopaminergic system [50], all brain structures functionally engaged in memory encoding and consolidation processes. Apart from the systems-level implications of these results for memory formation, they already demonstrated the important influence of local synaptic plasticity on activity propagation in brain-wide networks. Furthermore, the potentiation of neuronal responses, indicated by the steepened slope of the population excitatory postsynaptic potentials and the increased amplitude of the population spikes, correlated with the amplitude of the blood oxygen level-dependent signal, thus validating the usefulness of MRI for assessing global plasticity changes [51]. In contrast to detecting the effects elicited by noninvasive stimulation protocols only by means of their final parameters and comparing those to the corresponding datasets gathered prior to NIBS or rSS, a coupled on-going monitoring of stimulation effects is probably the more advantageous alternative. A multiparametric analysis of stimulation candidates, including noninvasive imaging data, may help establish treatment groups, defined as a homogenous sample of study participants that could benefit from personalized stimulation protocols. MRI techniques in particular lend themselves to this task, offering a high spatial resolution of the effect origin as well as being able to trace its spreading along brain-wide functional networks. What is more, connections between separate MRI-assessed parameters were drawn by Stagg and colleagues [53], who revealed an inverse correlation between GABA levels and resting state motor network connectivity. In turn, the higher amount of accessible details through noninvasive neuroimaging would assist with developing a more graded distinction between individuals in comparison with solely differentiating between responders and nonresponders, of whom the latter turned out to be less rare than previously supposed [7].

When refraining from a posteriori sample enrichments by excluding individuals who did not exhibit a response in the expected direction, the intersubject variability at hand puts forth two consecutive considerations. First, basic research endeavours to pinpoint the neurophysiological underpinnings of NIBS and rSS, insights about which would help to identify possible disturbing factors in trying to homogenize group results. Second, this homogenization would lend immediate assistance to the effective application of such treatments in clinical cases. Naturally, not all covariates are as simple to supervise and to control for as the participants' attention to the task [54], their serotonin levels [55,56"], or the presence of low doses of ethanol $[57,58]$, which were all shown to affect the elicitation of plasticity processes. In fact, identified genotypic markers for the expected effect of stimulation protocols, like the Val66Met polymorphism $\left[40^{-}\right]$, can only be registered and not regulated. Furthermore, different disease patterns, including major depression disorder [59], NMDAR encephalitis [23], and schizophrenia [60], are accompanied by a deficit of LTP-like plasticity. Nevertheless, knowledge concerning these influences can be used for the prediction of stimulation effects, which could still be considered a significant improvement to research proceeding along the lines of trial and error. At this point, the combination of NIBS and MRI techniques gains in importance, not only for the latter's usefulness in depicting a variety of different brain network properties, ranging from anatomical to neurochemical to functional, but also for the feasibility of continuously recording data in vivo during the ongoing stimulation period [61"]. Even the baseline functional connectivity, provided by fMRI, was successfully consulted to predict the efficiency of tDCS-induced modulations in terms of network connectivity in healthy individuals [33] as well as relative to analgesic effects in fibromyalgia patients [8]. 
Correspondingly, MRS measurements of neurochemicals in general and glutamatergic metabolites in particular are of predictive value, as the higher baseline levels of the latter are correlated with greater reductions in clinical pain scores following tDCS in fibromyalgia patients [8].

\section{CONCLUSION}

This review outlines that the claim of a consistent congruence between long-term synaptic plasticity and NIBS-induced effects is largely unfounded and leads to a deceptive terminology. All the more, the need for clarifying the neurophysiological underpinnings of the observed stimulation effects persists. This is not solely motivated by basic research interests but instead, a profound understanding would contribute to the customization of various NIBS protocols, which is of two-fold importance. First, the prediction of treatment results would allow to select only those individuals who can be reasonably expected to profit from the stimulation. Even though no long-term adverse effects are known for tDCS nor for related stimulation protocols, this approach might help to control for unnecessary expenditure of time and funds. Second, a categorization of individuals prior to the stimulus application would render an intervention into the distinct baseline state possible, thereby increasing the chances of a favourable response to the stimulation. In future years, stimulation protocols attuned to distinct treatment groups will probably be in rising demand where the first step will be a general homogenization of employed study parameters across research groups.

\section{Acknowledgements}

None.

\section{Financial support and sponsorship}

S.C. is supported by grants from the Spanish MINECO $B F U 2015-64380-C 2-1-R$ and from the EU Horizon 2020 Program 668863-SyBil-AA.

\section{Conflicts of interest}

There are no conflicts of interest.

\section{REFERENCES AND RECOMMENDED \\ READING}

Papers of particular interest, published within the annual period of review, have been highlighted as:

- of special interest

-1 of outstanding interest

1. Meinzer $M$, Jähnigen $S$, Copland $D A$, et al. Transcranial direct current stimulation over multiple days improves learning and maintenance of a novel vocabulary. Cortex 2014; 50:137-147.
2. Hsu W-Y, Zanto TP, Anguera JA, et al. Delayed enhancement of multitasking performance: effects of anodal transcranial direct current stimulation on the prefrontal cortex. Cortex 2015; 69:175-185.

3. Callan DE, Falcone B, Wada A, Parasuraman R. Simultaneous tDCS-fMRI

- identifies resting state networks correlated with visual search enhancement. Front Hum Neurosci 2016; 10:72.

The authors illustrate that resting state functional connectivity can be used to predict the improvement in a visual search task after tDCS. Transferring this observation to other areas of application should help to identify study participants, who would benefit from the stimulation, prior to tDCS.

4. Shirota Y, Hewitt M, Paulus W. Neuroscientists do not use noninvasive brain stimulation on themselves for neural enhancement. Brain Stimul 2014; 7:618-619.

5. Maslen H, Earp BD, Cohen Kadosh R, Savulescu J. Brain stimulation for treatment and enhancement in children: an ethical analysis. Front Hum Neurosci 2014; 8:953.

6. Brem A-K, Fried PJ, Horvath JC, et al. Is neuroenhancement by noninvasive brain stimulation a net zero-sum proposition? Neuroimage 2014; 85:10581068.

7. Wiethoff $\mathrm{S}$, Hamada M, Rothwell JC. Variability in response to transcranial direct current stimulation of the motor cortex. Brain Stimul 2014; 7:468-475.

8. Cummiford CM, Nascimento TD, Foerster BR, et al. Changes in resting state functional connectivity after repetitive transcranial direct current stimulation applied to motor cortex in fibromyalgia patients. Arthritis Res Ther 2016; 18:40.

9. Chen JL, Schlaug G. Increased resting state connectivity between ipsilesional motor cortex and contralesional premotor cortex after transcranial direct current stimulation with physical therapy. Sci Rep 2016; 6:23271.

10. Flöel A, Cohen LG. Contribution of noninvasive cortical stimulation to the study of memory functions. Brain Res Rev 2007; 53:250-259.

11. Ulm $L$, McMahon $K$, Copland $D$, et al. Neural mechanisms underlying perile-

- sional transcranial direct current stimulation in aphasia: a feasibility study. Front Hum Neurosci 2015; 9:550.

The proof-of-principle study demonstrates that tDCS can be applied to individually determined target regions, determined by peak activity during task-specific baseline fMRI.

12. Clapp WC, Zaehle T, Lutz K, et al. Effects of long-term potentiation in the human visual cortex: a functional magnetic resonance imaging study. Neuroreport 2005; 16:1977-1980.

13. Clapp WC, Hamm JP, Kirk IJ, Teyler TJ. Translating long-term potentiation from animals to humans: a novel method for noninvasive assessment of cortical plasticity. Biol Psychiatry 2012; 71:496-502.

14. Beste C, Dinse HR. Learning without training. Curr Biol 2013; 23:R489R499.

15. Marquez-Ruiz J, Leal-Campanario $R$, Sanchez-Campusano $R$, et al. Transcranial direct-current stimulation modulates synaptic mechanisms involved in associative learning in behaving rabbits. Proc Natl Acad Sci U S A 2012; 109:6710-6715.

16. Rohan JG, Carhuatanta KA, McInturf SM, et al. Modulating hippocampal masticity with in vivo brain stimulation. J Neurosci 2015; 35:12824-12832. The authors demonstrate that in-vivo tDCS in rats enhances plasticity at pre and postsynaptic sites, thus providing a direct link between tDCS and synaptic plasticity. PPF and long-term plasticity, with regard to slope and amplitude of field potentials, were enhanced in the hippocampus of acutely prepared brain slices.

17. Lahr J, Peter J, Bach M, et al. Heterogeneity of stimulus-specific response modification: an fMRI study on neuroplasticity. Front Hum Neurosci 2014; 8:695.

18. Davidson TW, Bolic M, Tremblay F. Predicting modulation in corticomotor excitability and in transcallosal inhibition in response to anodal transcranial direct current stimulation. Front Hum Neurosci 2016; 10:49.

19. Lahr J, Paßmann S, List J, et al. Effects of different analysis strategies on paired associative stimulation. A pooled data analysis from three research labs. PLoS One 2016; 11:e0154880.

20. López-Alonso V, Cheeran $B$, Rio-Rodriguez D, Fernández-del-Olmo M. Interindividual variability in response to noninvasive brain stimulation paradigms. Brain Stimul 2014; 7:372-380.

21. Strube W, Bunse $T$, Malchow $B$, Hasan A. Efficacy and interindividual

1. variability in motor cortex plasticity following anodal tDCS and paired-associative stimulation. Neural Plast 2015; 2015:1-10.

The study shows that the likelihood of being a nonresponder depends on the chosen NIBS protocol, with more participants responding to paired associative stimulation (PAS25) as compared with anodal tDCS. Additionally, it reported the predictive value of baseline intracortical facilitation for the efficacy of tDCS.

22. Bolzoni F, Bączyk M, Jankowska $E$. Subcortical effects of transcranial direct current stimulation in the rat: subcortical effects of tDCS. J Physiol 2013; 591:4027-4042.

23. Volz MS, Finke $C$, Harms $L$, et al. Altered paired associative stimulationinduced plasticity in NMDAR encephalitis. Ann Clin Transl Neurol 2016; 3:101-113.

24. Hilgenstock R, Weiss $T$, Huonker R, Witte OW. Behavioural and neurofunctional impact of transcranial direct current stimulation on somatosensory learning: tDCS and somatosensory learning. Hum Brain Mapp 2016; $37: 1277-1295$ 
25. Polania R, Nitsche MA, Paulus W. Modulating functional connectivity patterns and topological functional organization of the human brain with transcranial direct current stimulation. Hum Brain Mapp 2011; 32:1236-1249.

26. Wu D, Wang J, Yuan Y. Effects of transcranial direct current stimulation on naming and cortical excitability in stroke patients with aphasia. Neurosci Lett 2015; 589:115-120.

27. Meinzer $M$, Antonenko $D$, Lindenberg $R$, et al. Electrical brain stimulation improves cognitive performance by modulating functional connectivity and task-specific activation. J Neurosci 2012; 32:1859-1866.

28. Clemens $B$, Jung $S$, Mingoia $G$, et al. Influence of anodal transcranial direct current stimulation ( $t D C S$ ) over the right angular gyrus on brain activity during rest. PLoS One 2014; 9:e95984.

29. Keeser $D$, Meindl $T$, Bor $J$, et al. Prefrontal transcranial direct current stimulation changes connectivity of resting-state networks during fMRI. J Neurosci 2011; 31:15284-15293.

30. Peña-Gómez C, Sala-Lonch R, Junqué $C$, et al. Modulation of large-scale brain networks by transcranial direct current stimulation evidenced by resting-state functional MRI. Brain Stimul 2012; 5:252-263.

31. Krishnamurthy V, Gopinath $K$, Brown GS, Hampstead BM. Resting-state fMRI reveals enhanced functional connectivity in spatial navigation networks after transcranial direct current stimulation. Neurosci Lett 2015; 604:80-85.

32. Sehm B, Kipping J, Schäfer A, et al. A comparison between uni- and bilateral tDCS effects on functional connectivity of the human motor cortex. Front Hum Neurosci 2013; 7:183.

33. Polanía R, Paulus W, Nitsche MA. Reorganizing the intrinsic functional architecture of the human primary motor cortex during rest with non-invasive cortical stimulation. PLoS One 2012; 7:e30971.

34. Amadi $U$, llie A, Johansen-Berg H, Stagg CJ. Polarity-specific effects of motor transcranial direct current stimulation on fMRI resting state networks. Neuroimage 2014; 88:155-161.

35. Zheng $X$, Alsop DC, Schlaug G. Effects of transcranial direct current stimulation (tDCS) on human regional cerebral blood flow. Neuroimage 2011; 58:26-33.

36. Ranieri F, Podda MV, Riccardi E, et al. Modulation of LTP at rat hippocampal CA3-CA1 synapses by direct current stimulation. J Neurophysiol 2012; 107:1868-1880.

37. Monte-Silva K, Kuo M-F, Hessenthaler S, et al. Induction of late LTP-like plasticity in the human motor cortex by repeated non-invasive brain stimulation. Brain Stimul 2013; 6:424-432.

38. Fritsch $\mathrm{B}$, Reis J, Martinowich $\mathrm{K}$, et al. Direct current stimulation promotes BDNF-dependent synaptic plasticity: potential implications for motor learning. Neuron 2010; 66:198-204.

39. Podda MV, Cocco S, Mastrodonato A, et al. Anodal transcranial direct current

- stimulation boosts synaptic plasticity and memory in mice via epigenetic regulation of Bdnf expression. Sci Rep 2016; 6:22180.

The authors show that anodal tDCS affects the genetic expression of $B D N F$, which reflects late LTP processes.

40. Puri R, Hinder MR, Fujiyama $H$, et al. Duration-dependent effects of the BDNF

- Val66Met polymorphism on anodal tDCS induced motor cortex plasticity in older adults: a group and individual perspective. Front Aging Neurosci 2015; 7:107.

The article shows that the BDNFVal66Met polymorphism partially accounts for the variable responsiveness to tDCS of individuals, with Met carriers being more likely to show stimulation effects.

41. Kim S, Stephenson MC, Morris PG, Jackson SR. tDCS-induced alterations in GABA concentration within primary motor cortex predict motor learning and motor memory: a $7 \mathrm{~T}$ magnetic resonance spectroscopy study. Neuroimage 2014; 99:237-243.

42. Heba S, Puts NAJ, Kalisch T, et al. Local GABA concentration predicts

n- perceptual improvements after repetitive sensory stimulation in humans. Cereb Cortex 2016; 26:1295-1301.

The study indicates that the baseline GABA concentration of participants explained $\sim 60 \%$ of the variance in the gain of tactile perception from rSS. Hence, GABA levels assessed prior to stimulation possess a predictive power as to the stimulation outcome of individuals.

43. Bachtiar V, Near J, Johansen-Berg H, Stagg CJ. Modulation of GABA and

-1. resting state functional connectivity by transcranial direct current stimulation. Elife 2015; 4:e08789.

In this study, anodal tDCS decreased M1 GABA levels, whereas increasing functional connectivity in M1. However, no correlation substantiated between the two tDCS-induced changes, suggesting that both are driven by different underlying mechanisms, which tDCS affects differentially.
44. O'Shea J, Boudrias $\mathrm{M}-\mathrm{H}$, Stagg CJ, et al. Predicting behavioural response to TDCS in chronic motor stroke. Neuroimage 2014; 85:924-933

45. Clark VP, Coffman BA, Trumbo MC, Gasparovic C. Transcranial direct current stimulation (tDCS) produces localized and specific alterations in neurochemistry: a (1)H magnetic resonance spectroscopy study. Neurosci Lett 2011; 500:67-71.

46. Hunter MA, Coffman BA, Gasparovic C, et al. Baseline effects of transcranial

- direct current stimulation on glutamatergic neurotransmission and large-scale network connectivity. Brain Res 2015; 1594:92-107.

By combining $\mathrm{fMRI}$ and MRS for the surveillance of tDCS effects, the authors were able to show a positive correlation of combined glutamine and glutamate levels with network functional connectivity. This confirms the value of multimodal assessments of tDCS effects.

47. Errico F, Napolitano F, Squillace M, et al. Decreased levels of d-aspartate and NMDA in the prefrontal cortex and striatum of patients with schizophrenia. J Psychiatr Res 2013; 47:1432-1437.

48. Frankle WG, Cho RY, Prasad KM, et al. In vivo measurement of GABA

- transmission in healthy subjects and schizophrenia patients. Am J Psychiatry $2015 ; 172: 1148-1159$.

This is the first study to demonstrate an impaired GABA transmission in schizophrenia patients. Insights into the in-vivo GABA transmission can help explain the inconsistent findings of MRS GABA measures.

49. Hoy KE, Bailey NW, Arnold SL, Fitzgerald PB. The effect of transcranial direct

- current stimulation on gamma activity and working memory in schizophrenia. Psychiatry Res 2015; 228:191-196.

In this study, the application of tDCS enhanced the working memory of schizophrenia patients, presumably because of the restoration of gamma oscillations.

50. Canals S, Beyerlein M, Merkle H, Logothetis NK. Functional MRI evidence for LTP-induced neural network reorganization. Curr Biol 2009; 19:398403.

51. Alvarez-Salvado E, Pallares V, Moreno A, Canals S. Functional MRI of longterm potentiation: imaging network plasticity. Philos Trans R Soc B Biol Sci 2013; 369:20130152.

52. Moreno A, Morris RGM, Canals S. Frequency-dependent gating of hippocampal-neocortical interactions. Cereb Cortex 2016; 26:21052114.

53. Stagg CJ, Bachtiar V, Amadi U, et al. Local GABA concentration is related to network-level resting functional connectivity. Elife 2014; 3:e01465.

54. Klöppel S, Lauer E, Peter J, et al. LTP-like plasticity in the visual system and in the motor system appear related in young and healthy subjects. Front Hum Neurosci 2015; 9:506.

55. Nitsche MA, Kuo M-F, Karrasch R, et al. Serotonin affects transcranial direct current-induced neuroplasticity in humans. Biol Psychiatry 2009; 66:503508.

56. Kuo H-I, Paulus W, Batsikadze G, et al. Chronic enhancement of serotonin

- facilitates excitatory transcranial direct current stimulation-induced neuroplasticity. Neuropsychopharmacology 2016; 41:1223-1230.

The study highlights the impact of enhanced serotonin levels on tDCS effects and relates it to NMDAR-dependent plasticity processes.

57. Lücke $C$, Heidegger $T$, Röhner $M$, et al. Deleterious effects of a low amount of ethanol on LTP-like plasticity in human cortex. Neuropsychopharmacology 2014; 39:1508-1518.

58. Fuhl A, Müller-Dahlhaus F, Lücke $C$, et al. Low doses of ethanol enhance LTDlike plasticity in human motor cortex. Neuropsychopharmacology 2015; 40:2969-2980.

59. Kuhn M, Mainberger F, Feige $B$, et al. State-dependent partial occlusion of cortical LTP-like plasticity in major depression. Neuropsychopharmacology 2016; 41:1521-1529.

60. Hasan A, Nitsche MA, Rein B, et al. Dysfunctional long-term potentiation-like plasticity in schizophrenia revealed by transcranial direct current stimulation. Behav Brain Res 2011; 224:15-22.

61. Hone-Blanchet A, Edden RA, Fecteau S. Online effects of transcranial direct

- current stimulation in real time on human prefrontal and striatal metabolites. Biol Psychiatry 2015. [Epub ahead of print]

This is the first study to survey online tDCS effects by means of MRS, registering increased $\mathrm{N}$-acetylaspartate as well as combined glutamate and glutamine levels in response to tDCS. This approach is more sensitive to transient stimulation effects, which are not accessible by comparing pre and poststimulation effects. 